\title{
Теоретическое исследование генерации излучения волоконного лазера с каскадным усилением
}

\author{
С.А. Ефремов ${ }^{1}$, О.В. Штырина ${ }^{1,2}$, А.В. Иваненко ${ }^{1,3, *}$, \\ И.А. Яруткина ${ }^{1,2}$, А.С. Скидин ${ }^{1,2}$, М.П. Федорук ${ }^{1,2}$ \\ ${ }^{1}$ Новосибирский государственный университет \\ ${ }^{2}$ Институт вычислительных технологий СО РАН \\ ${ }^{3} \mathrm{OOO} \mathrm{«Техноскан-Лаб»,} \mathrm{г.} \mathrm{Новосибирск}$ \\ *E-mail: ivanenko.aleksey@gmail.com
}

DOI:10.31868/RFL2018.106-107

Современные лазерные системы с каскадным усилением позволяют получать нетривиальные характеристики излучения, а также позволяют реализовать электронно управляемые алгоритмы установки заданных режимов генерации [1,2]; так, наличие в резонаторе нескольких участков активных волокон применяется в лазерах с дисперсионным управлением, а также делает возможным достижение большего усиления излучения за счёт оптимального распределения усиления и потерь. Такое свойство может быть полезным в режиме импульсной генерации, где излишнее усиление ведет за собой развитие модуляционной неустойчивости и разрушение солитона.

Поскольку увеличение количества активных волокон влечет за собой увеличение количества степеней свободы в модели, описывающей систему, актуальным является вопрос о нахождении аналитических соотношений, которые позволяют снизить трудоёмкость получения численных результатов.

Для описания пространственно-временной динамики медленной огибающей внутри волокна традиционно используется неконсервативное нелинейное уравнение Шредингера:

$$
\frac{\partial A}{\partial z}=-i \frac{\beta_{2}}{2} \frac{\partial^{2} A}{\partial t^{2}}-i \gamma|A|^{2} A+\sigma A .
$$

Здесь $\beta_{2}$ и $\gamma$ обозначают коэффициенты дисперсии и нелинейности, соответственно. Выбор $\sigma$ определяется тем, в пассивной или активной среде моделируется динамика сигнала: $\sigma \equiv$ const $<0$ соответствует пассивному волокну, усиливающая среда, в свою очередь, описывается при $\sigma(z)=$ $g /\left(1+S(z) / S_{\text {sat }}\right)>0$. Для поиска режимов генерации с установившимся балансом энергии внутри резонатора будем использовать балансные уравнения, описывающие динамику средней мощности $S=\int|A|^{2} d t / T_{R}$ в усиливающем волокне:

$$
\frac{\partial S}{\partial z}=\frac{g}{1+S(z) / S_{\text {sat }}} S \Rightarrow S(0)=S_{\text {sat }} \frac{g L-\ln (G)}{G-1},
$$

где коэффициент насыщенного усиления $G=S(L) / S(0)$.

Любой лазер с каскадным усилением можно представить как пример кольцевого резонатора с $n$ усиливающими элементами и мультипликативным учетом потерь, как это показано на рисунке 1(а). Тогда внутрирезонаторная динамика средней мощности полностью определяется множествами коэффициентов усиления сигнала $\left\{1<G_{i}<\exp \left(g_{i} L_{i}\right)\right\}$ и потерь $\left\{R_{i}<1\right\}$. Для устойчивой генерации требуется равенство величины, обратной к полным потерям $\Sigma=\prod R_{i}$, и полного внутрирезонаторного усиления $G_{\text {total }}=\prod G_{i}$, то есть $G_{\text {total }}=1 / \Sigma$. В качестве начального приближения распределения усиления будем использовать последовательность коэффициентов $\left\{G_{i}{ }^{0}=G_{\text {total }}{ }^{1 / n}, 1 \leq i \leq n\right\}$.

Учитывая все вышеизложенное, построим итерационный процесс: 


$$
\left\{S_{i}(0)^{j}=S_{\text {sat }} \frac{g_{i} L_{i}-\ln \left(G_{i}{ }^{j}\right)}{G_{i}{ }^{j}-1}\right\},\left\{S_{i}\left(L_{i}\right)^{j}=S_{i+1}(0)^{j} / R_{i}\right\} \Rightarrow\left\{G_{i}^{j+1}=\frac{S_{i}\left(L_{i}\right)^{j}}{S_{i}(0)^{j}}\right\} .
$$

Данная система уравнений итерируется до выполнения условия с заданной погрешностью $\varepsilon: \quad \max _{1 \leq i \leq n}\left|G_{i}{ }^{j}-G_{i}{ }^{j+1}\right|<\varepsilon$.

В случае сильнонелинейной внутрирезонаторной динамики в представленном алгоритме может быть использована более общая модель, включающая зависимость от мощности накачки и фоновых потерь в активном волокне [3]. Алгоритм легко обобщается за счет интегрируемости эффективной двухуровневой модели.

На рис.1 приведены: схема конфигурации лазера (a), график внутрирезонаторной динамики средней мощности сигнала (b) и зависимость мощности излучения от количества активных элементов (c). В ходе расчетов использовались следующие параметры активного волокна [4]: $g_{i}=4.73$ дБ, $S_{s a t}=0.025 \mathrm{BT}, L_{i}=1$ м. Потери между усиливающими участками равны 0.3 дБ, а на участке с ответвителем выбраны равными 3.3 дБ. Так как коэффициент насыщенного усиления на каждом последующем участке активного волокна меньше предыдущего и в пределе стремится к единице, на графике 1(с) наблюдаем сходимость средней мощности излучения к некоторому оптимальному значению. Это позволяет утверждать, что при количестве усиливающих волокон в каскаде более нескольких десятков, увеличение энергии излучения осуществляется только за счет увеличения общей длины резонатора и пропорционально ей.
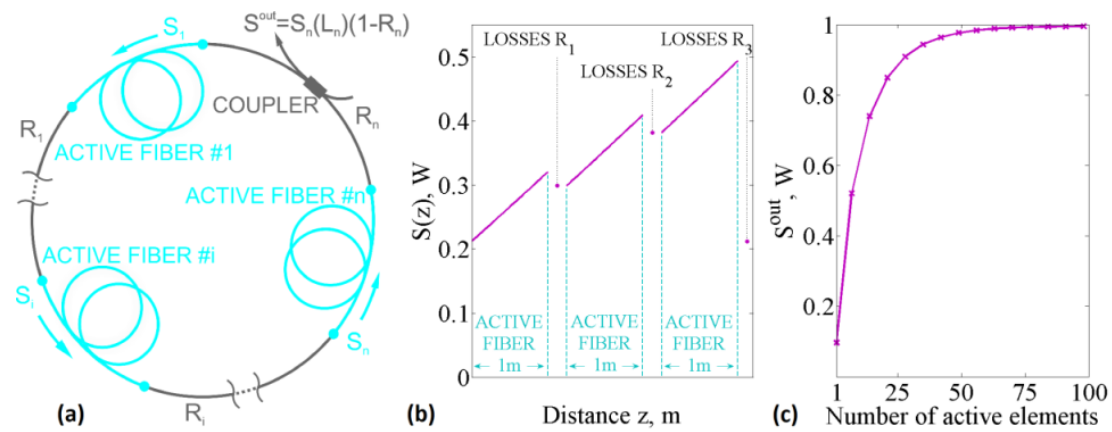

Рис. 1. Схема конфигурации лазера (а), внутрирезонаторная динамика средней мощности сигнала $S$ для $n=3(\mathrm{~b})$, зависимость мощности излучения $S^{\text {out }}$ от количества активных элементов (c).

Таким образом, построен итерационный алгоритм для определения распределения усиления внутри полости резонатора с произвольным количеством различных активных волокон. Данные результаты могут быть полезны для оптимизации характеристик излучения волоконных лазеров. Работа O.В. Штыриной (теоретический анализ) была выполнена при поддержке Российского фонда фундаментальных исследований (проект РФФИ 18-01-00775), работа А.В. Иваненко (обсуждение результатов) была выполнена при поддержке проекта РФФИ 16-32-60160.

\section{Литература}

[1] S.Smirnov, S.Kobtsev et al. Opt. Lett., 42(9), 1732-1735 (2017).

[2] S. Kobtsev, A. Ivanenko et al. Las. Phys. Lett., 15(4), 045102 (2018).

[3] C. Barnard, P. Myslinski et al. IEEE J. Quantum Electron. 30, 1817-1830 (1994).

[4] O. V. Shtyrina, A. V. Ivanenko et al. J. Opt. Soc. Am. B, 34(2), 227-231 (2017). 